\title{
Fundamentos microestructurales de la anisotropía resistente en aceros fuertemente trefilados
}

\author{
J. Toribio ${ }^{(*)}$, E. Ovejero ${ }^{(*)}$ y M. Toledano $(*)$ \\ Resumen Se propone una explicación del comportamiento anisótropo en fractura que exhiben los aceros fuerte- \\ mente trefilados sobre la base de consideraciones micromecánicas: el cambio de dirección de fisura- \\ ción puede explicarse por la microestructura perlítica orientada de estos aceros. Aparte de las colo- \\ nias perlíticas tipo (más o menos orientadas), a veces puede observarse una pseudocolonia perlítica \\ excepcional, extremadamente esbelta y alineada en la dirección del trefilado, lo que hace de ella un \\ camino preferente de fractura con resistencia local mínima. \\ Palabras clave: Aceros perlíticos. Trefilado progresivo. Microestructura del acero. Anisotropía \\ resistente.

\section{Microstructural basis of anisotropic fracture behaviour of heavily drawn steels}

\begin{abstract}
An explanation of the anisotropic fracture behaviour of heavily drawn steels is proposed on the basis of micromechanical considerations: the change in crack propagation direction can be explained by the oriented pearlitic microstructure of the steels. Apart from the standard pearlitic colonies (more or less oriented), sometimes an exceptional pearlitic pseudocolony can be observed, extremely slender and aligned in the drawing direction, which makes it a preferential fracture path with minimum local
\end{abstract} resistance.

Keywords: Pearlitic steels. Progressive cold drawing. Steel microstructure. Resistance anisotropy

\section{INTRODUCCIÓN}

La técnica del hormigón pretensado en ingeniería civil requiere el uso de aceros perlíticos fuertemente trefilados en forma de alambres de acero de pretensado que han sobrellevado deformaciones plásticas muy intensas. El proceso de fabricación consiste en el trefilado progresivo en varias etapas, para aumentar el límite elástico, mediante un mecanismo de endurecimiento por deformación, produciendo de este modo cambios microestructurales en el material en forma de orientación de la microestructura perlítica ( 1 y 2 ), lo que podría explicar el comportamiento anisótropo observado en la fractura de este tipo de aceros en aire ( 3 y 4 ) y en ambientes agresivos (4-6).

(*) Dpto. de Ciencia de Materiales. Univ. de La Coruña. E.T.S.I. Caminos, Campus de Elviña, 15192-La Coruña (España).
Este artículo trata de establecer bases microestructurales para el citado comportamiento anisótropo en fractura de los aceros fuertemente trefilados (es decir, los aceros de las etapas finales de la cadena de fabricación). Para ello, se analiza la relación entre las peculiaridades microestructurales inducidas por el trefilado intenso y el cambio repentino en la dirección de propagación de fisuras que aparece en dichos aceros cuando una fisura llega a ser crítica como consecuencia de un micromecanismo de fractura especial consistente en la fisuración por esfuerzo cortante de las láminas de perlita.

\section{MATERIALES UTILIZADOS}

En este trabajo se utilizaron aceros eutectoides de alta resistencia suministrados por EMESA TREFILERIA (La Coruña), procedentes de todas las etapas del proceso de fabricación mediante trefilado 
progresivo, desde el alambrón laminado en caliente (acero A0 no trefilado en absoluto) hasta el alambre de pretensado (acero A6 que ha sufrido seis pasos de trefilado y representa el producto final comercial). El diámetro de los alambres se reduce progresivamente a lo largo del proceso desde $12 \mathrm{~mm}$ (alambrón) hasta $7 \mathrm{~mm}$ (trefilado) y el límite elástico aumenta desde $686 \mathrm{MPa}$ (alambrón) hasta 1.506 MPa (trefilado). Esta mejora de propiedades mecánicas (incremento del límite elástico) es precisamente el objetivo - beneficioso- del trefilado. Sin embargo, las consecuencias de este método de fabricación, desde el punto de vista del comportamiento en fractura, no se conocen todavía en su totalidad.

\section{ESTUDIO MICROESTRUCTURAL}

El primer nivel microestructural de los aceros es la colonia de perlita como un conjunto de láminas de ferrita y cementita con la misma orientación. Considerando la colonia de perlita como un elipsoide, los resultados del análisis metalográfico (7) revelaron que el primer efecto del trefilado es la orientación progresiva de la colonia con su eje principal aproximándose al eje del alambre o dirección de trefilado, y este efecto parece ser predominante en los primeros estadios de la cadena de fabricación. El segundo efecto del estirado en frío consiste en un aumento de esbeltez de las colonias de perlita, con un claro alargamiento del eje mayor o principal y un moderado acortamiento del eje secundario (perpendicular al anterior), y este efecto parece ser predominante en los últimos pasos del proceso. Ambos efectos tienden a incrementar la densidad de apilamiento transversal a medida que el trefilado tiene lugar, lo que resulta especialmente intenso para los aceros fuertemente trefilados (últimos pasos del proceso de fabricación).

El segundo nivel microestructural es la estructura laminar perlítica. Con respecto a la misma, el trefilado produce una disminución progresiva del espaciado interlaminar y una orientación gradual de las láminas de perlita que tienden a alinearse en la dirección del eje del alambre o dirección de trefilado (8). De este modo, la microestructura evoluciona hacia estructuras más densas y orientadas a medida que la deformación plástica producida por el trefilado aumenta.

Las características descritas en los párrafos anteriores representan la tendencia general de la evolución microestructural producida por el estirado en frío de los aceros. Sin embargo, existen algunas pseudocolonias perlíticas excepcionales muy esbeltas, alineadas en la dirección del trefilado y cuyo espaciado interlaminar local es claramente anómalo (especialmente elevado) en comparación con el espaciado medio (o global) del acero en cuestión. Además de lo anterior, se pueden encontrar evidencias de microfisuras inducidas por esfuerzo cortante creadas durante el proceso de fabricación en dichas colonias especiales o anómalas.

\section{COMPORTAMIENTO EN FRACTURA}

Para analizar el comportamiento en fractura de los aceros con diferentes grados de trefilado se utilizaron muestras cilíndricas fisuradas obtenidas de los alambres de acero. La pre-fisura se produjo mediante fatiga en dirección axial, y los alambres fisurados fueron sometidos a un proceso de carga monótona de tracción hasta la fractura final. La figura $1 a$ muestra el aspecto de la fractura en aceros con diferentes grados de trefilado. El hecho experimental fundamental observado en los aceros con nivel de trefilado más intenso es el cambio en la dirección de propagación de la fisura, de manera que ésta tiende a propagarse paralelamente a la dirección de trefilado o eje del alambre, lo que representa una propagación en modo mixto con una desviación de casi $90^{\circ}$ con respecto a la propagación inicial en modo I en dirección transversal (perpendicular al eje del alambre). Mientras que el alambrón laminado en caliente (A0 no trefilado en absoluto) y los aceros débilmente trefilados (A1, A2 y A3) exhiben un comportamiento isótropo o cuasiisótropo en fractura (totalmente isótropo en el caso del alambrón), los aceros fuertemente trefilados (A4, A5 y A6) muestran un comportamiento en fractura claramente anisótropo asociado con una desviación de unos $90^{\circ}$ con respecto al plano inicial (escalón de propagación) y un crecimiento posterior según una dirección próxima a la inicial (a $20-30^{\circ}$ de la misma). Como se muestra esquemáticamente en la figura $1 b$, este escalón a $90^{\circ}$ aparece a cierta distancia $x_{S}$ medida desde el extremo de la fisura de fatiga, y esta distancia disminuye cuando el grado de trefilado del acero aumenta, es decir, el escalón se aproxima a la fisura de fatiga a medida que el nivel de trefilado es más intenso, y así, en el alambre de pretensado totalmente trefilado (A6), el escalón se localiza exactamente en el borde de la pre-fisura de fatiga $\left(x_{S}=0\right)$. La apariencia microscópica de dicho escalón al microscopio electrónico de barrido aparece en la figura $1 c$, y recuerda un tipo de fractura por esfuerzo cortante. Aparte del escalón, las superficies de fractura son del tipo crecimiento y coalescencia de huecos $(\mathrm{CCH})$ antes del escalón (cuando $x_{S}>0$ ) y $\mathrm{CCH}$ con algunas facetas de clivaje $(C)$ después del mismo (Fig. $1 b$ ). De este modo, la distancia $x_{S}$ representa la extensión de la propagación de fisura en modo I por $\mathrm{CCH}$ antes de 


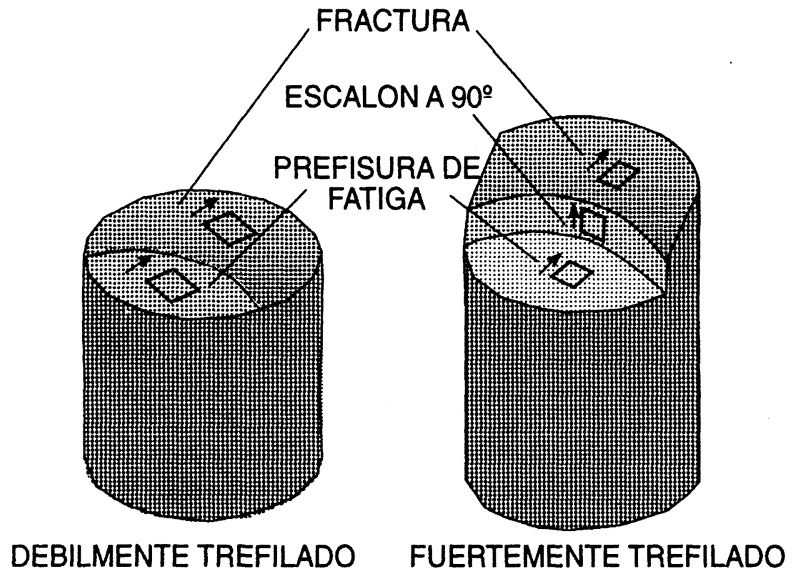

(a)

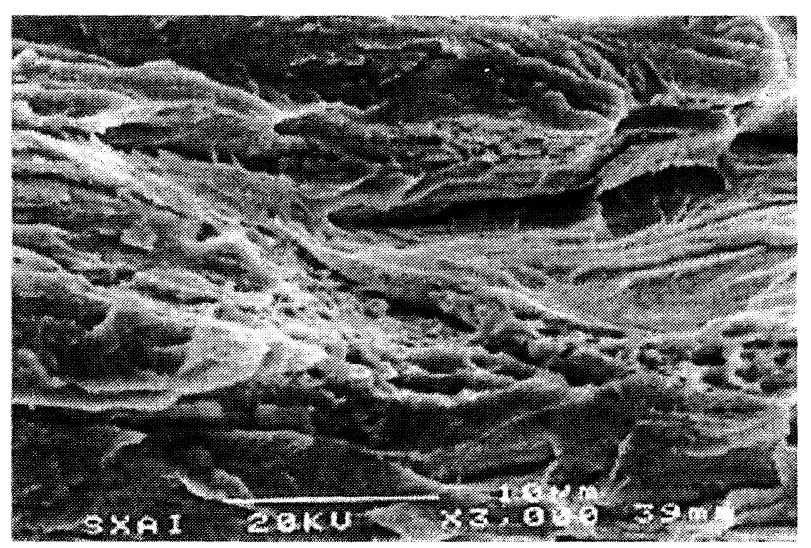

(c)

la propagación en modo mixto producida por el escalón a $90^{\circ}$, y esta extensión simplemente no existe en el caso del acero más fuertemente trefilado A6.

\section{DISCUSIÓN}

El comportamiento anisótropo descrito en los aceros fuertemente trefilados, consistente en un cambio en la dirección de propagación de la fisura - con respecto al plano inicial de propagación perpendicular al eje del alambre- puede explicarse por medio de consideraciones de índole micromecánica, sobre la base de la microestructura perlítica orientada de los aceros. La figura $2 a$ muestra una sección longitudinal de un acero fuertemente trefilado. Aparte de las colonias perlíticas tipo (más o menos orientadas), puede observarse una pseudocolonia perlítica excepcional, extremadamente esbelta, alineada cuasi-paralela al eje del alambre, y con un espaciado interlaminar local muy grande, debido al hecho de que las láminas de cementita no se han orientado según el eje del alambre y, en algunos casos, se encuentran pre-fracturadas por esfuerzo cor-

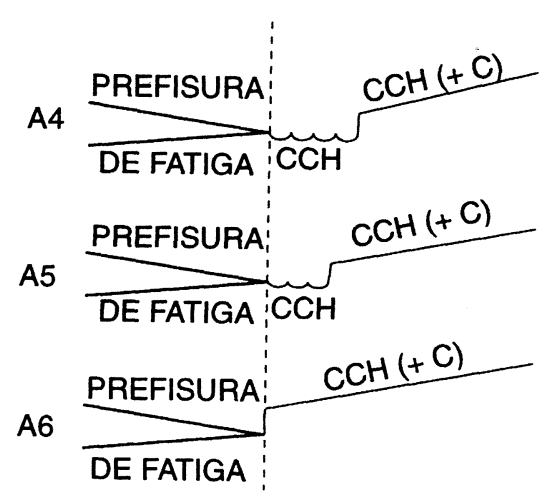

(b)

FIG. 1.- Fractura de los alambres de acero perlítico: a) Aspecto de la fractura en aceros con distintos grados de trefilado; $b$ ) Perfiles de propagación de la fisura en aceros fuertemente trefilados; $c$ ) Micrografía del escalón a $90^{\circ}$.

FIG. 1.- Fracture of pearlitic steel wires: a) View of the fracture in steels with different cold drawing degree; b) Propagation profiles of the crack in heavily cold drawn steels; c) Micrograph of the step at $90^{\circ}$.

tante durante el proceso de fabricación. Esta característica hace de ellas caminos preferentes de fractura con resistencia local mínima. Así pues, las pseudocolonias perlíticas actúan como precursoras de la micro-fisuración local, y su presencia podría explicar el escalón a $90^{\circ}$ observado en el camino de fractura de aceros fuertemente trefilados. La figura $2 b$ ofrece un esquema que muestra la formación del escalón a $90^{\circ}$ cuando la macro-fisura (la fisura de fatiga más la propagación inicial en modo I) alcanza la ubicación de la pseudocolonia perlítica, y en ese momento las láminas de perlita se fracturan mediante un mecanismo de fisuración por esfuerzo cortante (Fig. 2c) de acuerdo con el modelo propuesto por Miller y Smith (9), lo que además es consistente con la apariencia microscópica de fractura por cortante en el escalón a $90^{\circ}$ (Fig. 1c).

Finalmente, el micromecanismo de fractura propuesto puede explicar otro hecho experimental: la distancia $x_{S}$ entre la fisura de fatiga y el escalón (es decir, la longitud de propagación en modo I) es una función decreciente del grado de trefilado. Esto puede ser consecuencia de la frecuencia de aparición de pseudocolonias perlíticas que es más alta a 

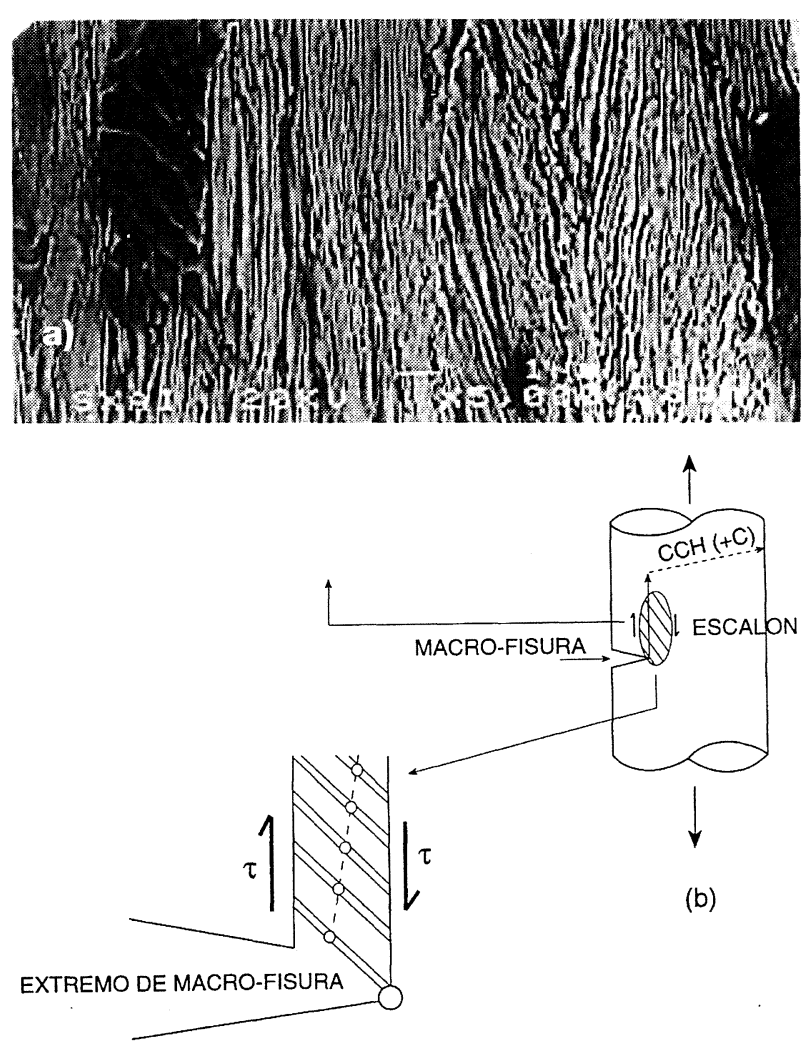

(c)

FIG. 2.- Microestructura y micromecanismos de fractura: a) Pseudocolonia perlítica esbelta en aceros fuertemente trefilados; $b$ ) Esquema que muestra la formación del escalón a $90^{\circ} ; \mathrm{c}$ ) Micromecanismo de fractura mediante fisuración de la perlita por esfuerzo cortante en la pseudocolonia, de acuerdo con el modelo propuesto por Miller y Smith (1970).

FIG. 2.- Microstructure and micromechanism of fracture: a) Pearlitic pseudocolony slender in heavily cold drawn steels; b) Scheme showing the formation of the step at $90^{\circ}$; c) Fracture micromechanism by means of pearlite cracking due to shearing stress on the pseudocolony, in agreement with the model proposed by Miller and Smith (1970).

medida que el trefilado se hace más intenso, es decir, la distancia media entre pseudocolonias es función decreciente del grado de trefilado. En aceros fuertemente trefilados, la probabilidad de cambio de dirección de propagación es realmente alta y, así, la macro-fisura se propaga en modo I hasta que la primera pseudocolonia especial aparece en la ruta de avance de la fisura y en ese momento se produce la propagación en modo mixto con aparición del escalón a $90^{\circ}$.

\section{CONCLUSIONES}

Se ha propuesto una explicación micromecánica del comportamiento anisótropo en fractura que exhiben los aceros perlíticos fuertemente trefilados, y que podría deberse a la presencia de pseudocolonias perlíticas muy esbeltas, cuasi-paralelas al eje del alambre y con espaciado interlaminar excesivo. Estas colonias, cuya frecuencia de aparición es mayor a medida que el trefilado es más intenso, explicarían la aparición de un escalón de propagación en el camino de fractura de los aceros, cuando la macrofisura alcanza la pseudocolonia y en ésta se alcanza la situación crítica debido a la fractura por cortante de las láminas de perlita anómalamente distribuidas.

\section{Agradecimiento}

Los autores desean hacer constar su agradecimiento por la financiación recibida a la CICYT (Ref. MAT97-0442) y a la Xunta de Galicia (Refs. XUGA 11801B95 y XUGA 11802B97). También agradecen a EMESA TREFILERIA S.A. el suministro del acero utilizado en el programa experimental.

\section{REFERENCIAS}

(1) Embury, J.D. y Fisher, R.M. Acta Metall. 14, 1966: 147159.

(2) LANGFORD, G. Metall. Trans. 1, 1970: 465-477.

(3) Astiz, M.A., Valiente, A., Elices, M. y Bui, H.D. Life Assessment of Dynamically Loaded Materials and Structures-ECF5 (Ed. por L.O. Faria), EMAS, Warley, 1984: 385393.

(4) Lancha, A.M. Tesis Doctoral, Universidad Complutense de Madrid (España), 1987.

(5) Cherry, B.W. y Price, S.M. Corros. Sci. 20, 1980: 11631184.

(6) Sarafianos, N. J. Mater. Sci. Lett. 8, 1989: 1486-1488.

(7) Toribio, J. y Ovejero, E. Mater. Sci. Eng., A234-236, 1997: 579-582.

(8) Toribio, J. y Ovejero, E. Mech. Time Dependent Mater. (Pendiente de publicación).

(9) Miller, L.E. y Smith, G.C. J. Iron Steel Inst. 208, 1970: 998-1005. 